\title{
EL CUESTIONARIO DE ANÁLISIS CLÍNICO (CAQ): FIABILIDAD Y VALOR DISCRIMINANTE EN UNA MUESTRA CLÍNICA
}

\author{
MARIA FORNS ${ }^{1}$, JUAN A. AMADOR ${ }^{1}$, JUDIT ABAD ${ }^{1}$ Y BERNARDÍ MARTORELL ${ }^{2}$ \\ ${ }^{1}$ Universidad de Barcelona \\ ${ }^{2}$ Servicio de Psiquiatría, Hospital de Sant Pau, Barcelona
}

(Recibido el 9 de enero de 1998)

\begin{abstract}
El Cuestionario de Análisis Clínico (CAQ) fue diseñado por Krug (1980) para analizar conductas psicopatológicas, como complemento clínico del test factorial 16PF de Cattell (1975). En España se han llevado a cabo pocas investigaciones en población psiquiátrica mediante el CAQ. El objetivo del presente trabajo es analizar la consistencia interna y el poder discriminante del CAQ en población clínica. La prueba de CAQ fue administrada a 250 pacientes procedentes del Dispensario de Psiquiatría del Hospital de St. Pau de Barcelona con edades comprendidas entre 18 y 65 años. Los sujetos fueron diagnosticados mediante criterios del DSM-III-R y agrupados en cuatro categorías. El valor medio del coeficiente alfa es satisfactorio mientras que el poder discriminante resulta moderado.

Palabras clave: Cuestionario de Análisis Clínico, fiabilidad, valor discriminante, psicopatología.

\section{Clinical Analysis Questionnaire (CAQ): Reliability and discriminant value in a clinical sample}

Krug's Clinical Analysis Questionnaire "CAQ" (Krug, 1980) was designed to analyze pathological behaviors to complement the source traits of personality offered by Cattell's 16PF. Very few clinical studies using CAQ have been made in Spain. The aim of this study was to analyze the internal consistency and the discriminant power of the CAQ in a clinical population. The CAQ was administered to 250 psychopathological subjects, aged 18 to 65 , classified according to the DSM-III-R criteria and organized in four groups. The patients were obtained from Psychiatric Department of St. Pau Hospital in Barcelona. The median alpha coefficient is satisfactory and the discriminant power found is moderate.
\end{abstract}

Key words: Clinical Analysis Questionnaire, reliability, discriminant value, psychopathology.

\section{INTRODUCCIÓN}

\section{El Cuestionario de Análisis Clínico} (CAQ; Krug, 1980) es un instrumento de evaluación que complementa al Cuestionario factorial de personalidad 16PF (Cattell, 1975) y se emplea para identificar conductas psicopatológicas.

Delhees y Cattell (1971) y Cattell (1973) trataron de identificar factores de

\footnotetext{
Correspondencia: Marfa Forns, Departamento de Personalidad, Evaluación y Tratamiento Psicológicos, Universidad de Barcelona, Passeig de la Vall d'Hebron 171, Edificio Ponent, 08035 Barcelona.
}

personalidad y de psicopatología que pudieran caracterizar la conducta de población clínica. Mediante estudios factoriales de los items del 16PF y del MMPI obtuvieron confirmación de las 16 escalas de personalidad del 16PF y de otros 12 factores de psicopatología. Cattell y Bjerstedt (1967) encontraron que la depresión no era factorialmente unidimensional, sino que podía desglosarse en siete factores, que configuran las siete primeras escalas presentadas en el CAQ. Tales escalas de contenido depresivo son distintas de los factores de personalidad derivados en el 16PF y han sido identifi- 
cadas con las denominaciones de Hipocondríasis, Depresion-suicida, Agitación, Depresión-ansiosa, Depresión baja-energía, Culpa-resentimiento y Apatía-retirada. Asimismo, mediante análisis factorial conjunto del MMPI y del 16 PF, Cattell y Bolton (1969), identificaron otros cinco factores, que tampoco se sobreponían con las escalas del 16 PF y que eran factorialmente independientes de los siete factores de tipo depresivo previamente identificados. Estos cinco factores son los que configuran las cinco últimas escalas del CAQ (Cattell, 1973), las cuales, a juicio de Krug y Cattell (1980, pág. 2) miden «rasgos» psicopatológicos ("psychopathological traits"). El contenido de estos 5 factores es identificado con las denominaciones de Paranoia, Desviación psicopática, Esquizofrenia, Psicastenia y Desajuste psicológico. La adaptación española del CAQ (Krug, 1987/1994) comprende el conjunto de estas 12 escalas clínicas.

Los estudios realizados con este instrumento se centran, básicamente, en el análisis psicométrico y factorial del test, tomando como referencia la población normal adulta (Schwartz, 1976; Kameoka, 1986; Boyle, 1987; Montag y Birenbaum, 1988; Sirigatti, Stefanile y Giannetti, 1990) y adolescente (Martorell, Amador y Forns, 1994; Abad, Amador, Forns y Martorell, 1996). Estos trabajos identifican un variado número de factores de segundo orden, con pesos explicativos diferentes, y con denominaciones variadas. La escasa coincidencia entre los factores detectados responde, a nuestro juicio, a dos hechos. Por una parte, los análisis factoriales del CAQ se han realizado conjuntamente con distintas pruebas que analizan rasgos de personalidad o conductas patológicas, cuyo contenido es probablemente poco homogéneo; por otra, los autores han ofrecido diferentes interpretaciones de normalidad o de psicopatología a los factores resultantes. Las investigaciones sobre la estructura factorial de primer orden del CAQ son escasas. Abad et al. (1996) presentan un análisis factorial de primer orden con población adolescente, escolarizada en enseñanza secundaria. Este análisis, que se refiere a población normal, genera 10 factores que ponen de relieve alteraciones de la adaptación emocional y social, más que conductas psicopatológicas.

Pocos estudios se han referido a la calidad clínica del test, a pesar de que el objetivo de la prueba reside precisamente en la identificación de características psicopatológicas. Marcos y Carné (1988) con una muestra de 30 sujetos diagnosticados de agorafobia obtienen un decatipo elevado en la escala de Psicastenia (As) y atribuyen esta elevación al contenido ansioso expresado en los ítems de esta escala. Carpenter (1995) se propuso identificar el perfil clínico y de personalidad de sujetos alcohólicos. A partir de un total de 277 sujetos, configuró cuatro grupos, atendiendo a la presencia/ausencia de alcoholismo en los sujetos y sus progenitores: sujetos alcohólicos versus no-alcohólicos y ascendencia familiar directa de alcoholismo versus no-alcoholismo. El grupo de sujetos alcohólicos con progenitores alcohólicos, aunque no mostraban un perfil definido, presentaban mayores elevaciones en múltiples escalas del CAQ. Martorell, Forns, Amador y Abad (1995) han analizado el perfil clínico obtenido por 152 sujetos procedentes de consulta psiquiátrica, diagnosticados utilizando los criterios del DSMIII-R (APA, 1987). Estos autores sugieren que el uso de decatipos no permite establecer la necesaria diferenciación entre los pacientes que obtienen puntuaciones brutas elevadas. Para solventar esta dificultad proponen el uso de las notas T, tal como ya fue sugerido por el propio Krug en 1989. Martorell et al. (1995) concluyen que el CAQ es útil para la detección de malestar psicopatológico, aunque su valor para establecer diag- 
nósticos diferenciales parece ser bastante escaso.

Los pocos trabajos que abordan el tema de la fiabilidad del CAQ, han sido llevados a cabo en población no clínica. Krug (1980) obtuvo unos valores promedio de 0,80 para la fiabilidad test-retest y de 0,71 para la consistencia interna ( $\alpha$ de Cronbach). En la adaptación española del test, realizada por la casa TEA, se ofrece un coeficiente alfa promedio de 0,51 (Krug, 1987-1994).

El objetivo de este trabajo es analizar la consistencia interna ( $\alpha$ de Cronbach) del CAQ en población clínica y el poder discriminativo de las escalas del CAQ para diferenciar entre grupos clínicos establecidos según criterios del DSM-III-R.

\section{MÉTODO}

\section{Sujetos}

La muestra está formada por 250 pacientes psiquiátricos (113 varones, 137 mujeres), de edades comprendidas entre 18 y 65 años, procedentes del ambulatorio del Servicio de Psiquiatría del Hospital Sant Pau de Barcelona.

El diagnóstico había sido establecido, con fines asistenciales, por el equipo clínico responsable de cada enfermo (psicólogo y psiquiatra), a partir de los datos obtenidos en la anamnesis, las entrevistas clínicas, test cognitivos y escalas psicopatológicas (MMPI), según los criterios del DSM-III-R ${ }^{1}$. Las pruebas psicológicas que configuran la batería diagnóstica eran aplicadas en el momento en que el sujeto estaba en condiciones para hacerlo. Cada paciente recibía el tratamiento psicofarmacológico y/o psicoterapéutico que el equipo consideraba pertinente.

\footnotetext{
${ }^{1}$ Los sujetos que componen la muestra han sido explorados a partir del año 1993, lo cual nos ha obligado a mantener, para este estudio, los criterios diagnósticos del DSM-III-R vigentes en aquel momento.
}

La muestra ha sido reclutada entre los años 1993 y 1996 . Una vez establecido el diagnóstico y durante el tratamiento, se invitaba a los pacientes a contestar el protocolo del CAQ, de forma voluntaria. Se excluyeron los sujetos que presentaban niveles intelectuales bajos (CIT<85), trastornos orgánicos y/o estados confusionales y aquellos cuyos diagnósticos fueran mixtos o aplazados.

Se configuraron cuatro grupos. El primero fue caracterizado por conductas y trastornos de contenido depresivo, incluyendo sujetos con Depresión Mayor y Distimia (Grupo 1= Depresión). En el segundo grupo se incluyeron las alteraciones psicóticas: sujetos afectos de esquizofrenia, esquizofreniformes y delirantes (Grupo 2 = Esquizofrenia). En el tercer grupo se recogieron las manifestaciones clínicas de la ansiedad, incluyendo a sujetos con trastornos de ansiedad (ansiedad generalizada, fóbicos, obsesivo-compulsivos, ataques de pánico) además de sujetos con trastornos somatomorfes y trastornos adaptativos con reacción ansiosa (Grupo $3=$ Ansiedad). Finalmente el cuarto grupo (Grupo 4 = Trastornos de Personalidad) estaba caracterizado por la conducta de impulsividad e incluía sujetos clasificados en el cluster B del eje II (Trastorno límite, Narcisista y/o Antisocial).

La composición de la muestra especificada según sexo, edad y grupo clínico se recoge en la Tabla 1.

\section{Instrumentos y procedimiento}

Se ha utilizado el Cuestionario de Análisis Clínico (CAQ) de Krug (1980) en la versión ofrecida por TEA (Krug, 19871994).

La prueba se administró a cada sujeto individualmente, siguiendo las consignas del manual. Se eligió el momento de aplicación en el que, según la naturaleza de la 
Tabla 1. Composición de la muestra

\begin{tabular}{lccccc}
\hline & \multicolumn{2}{c}{ SEXO } & \multicolumn{3}{c}{ EDAD } \\
\cline { 2 - 6 } GRUPOS CLÍNICOS & VARONES & MUJERES & $<25$ años & 25 a 40 años & $>40$ años \\
\hline Depresivos & 21 & 41 & 10 & 19 & 33 \\
Esquizofrénicos & $(19 \%)$ & $(30 \%)$ & $(14,5 \%)$ & $(20,2 \%)$ & $(37,9 \%)$ \\
& 33 & 36 & 27 & 28 & 14 \\
Ansiosos & $(29 \%)$ & $(26 \%)$ & $(39,1 \%)$ & $(29,8 \%)$ & $(16,1 \%)$ \\
& 37 & 41 & 10 & 35 & 33 \\
T. personalidad & $(33 \%)$ & $(30 \%)$ & $(14,5 \%)$ & $(37,2 \%)$ & $(37,9 \%)$ \\
& 22 & 19 & 22 & 12 & 7 \\
& $(19 \%)$ & $(14 \%)$ & $(31,9 \%)$ & $(12,8 \%)$ & $(8,0 \%)$ \\
\hline
\end{tabular}

alteración, cada sujeto podía adaptarse a las exigencias de un autoinforme (comprender y responder de forma pertinente), aunque se dispensaron ayudas en la lectura y clarificación de los enunciados cuando fueron requeridas. En la mayoría de casos la aplicación ha sido posterior al establecimiento del diagnóstico clínico, durante el tratamiento farmacológico y psicológico, y próxima a la fase de remisión.

\section{Análisis de datos}

Los datos han sido tratados mediante el paquete estadístico SPSS-PC+. Los análisis abarcan descriptivos básicos, coeficiente de consistencia interna ( $\alpha$ de Cronbach), análisis factorial de la varianza con contrastes Scheffeé y análisis discriminante.

\section{RESULTADOS}

La Tabla 2 presenta la puntuaciones medias y las desviaciones típicas de las escalas del CAQ para los cuatro grupos clínicos establecidos. También se incluyen los valores medios y la dispersión de las puntuaciones para la muestra de sujetos adultos normales. Estos últimos datos se

Tabla 2. Medias y dispersiones de las puntuaciones directas en las escalas del CAQ para los cuatro grupos clínicos y población normal

\begin{tabular}{|c|c|c|c|c|c|c|c|c|c|c|}
\hline \multirow[t]{2}{*}{$\begin{array}{l}\text { ESCALAS } \\
\text { DEL CAQ }\end{array}$} & \multicolumn{2}{|c|}{$\begin{array}{l}\text { DEPRESIVOS } \\
\quad(\mathrm{N}=62)\end{array}$} & \multicolumn{2}{|c|}{$\begin{array}{l}\text { ESQUIZOFRÉNICOS } \\
(\mathrm{N}=69)\end{array}$} & \multicolumn{2}{|c|}{$\begin{array}{l}\text { ANSIOSOS } \\
(\mathrm{N}=78)\end{array}$} & \multicolumn{2}{|c|}{$\begin{array}{l}\text { TRASTORNOS DE } \\
\text { PERSONALIDAD } \\
(\mathrm{N}=41)\end{array}$} & \multicolumn{2}{|c|}{$\begin{array}{c}\text { GRUPO } \\
\text { NORMATIVO } \\
(\mathrm{N}=2,971)\end{array}$} \\
\hline & $\mathrm{M}$ & SD & $\mathrm{M}$ & $S D$ & M & $S D$ & $\mathbf{M}$ & SD & $\mathrm{M}$ & $S D$ \\
\hline D1 & 12,80 & 5,96 & 8,91 & 5,53 & 12,79 & 5,29 & 9,02 & 4,32 & 1,81 & 2,27 \\
\hline D2 & 12,35 & 7,23 & 8,94 & 5,87 & 10,08 & 6,17 & 11,10 & 5,48 & 1,88 & 2,71 \\
\hline D3 & 8,00 & 3,15 & 10,11 & 2,95 & 8,68 & 2,94 & 11,71 & 3,06 & 10,47 & 3,13 \\
\hline D4 & 11,96 & 4,34 & 9,96 & 4,13 & 10,88 & 4,03 & 9,95 & 3,92 & 6,09 & 3,33 \\
\hline D5 & 15,45 & 6,23 & 11,36 & 5,99 & 15,56 & 5,59 & 13,00 & 5,40 & 4,42 & 3,49 \\
\hline D6 & 13,80 & 5,88 & 11,11 & 5,82 & 13,60 & 5,10 & 14,29 & 4,95 & 7,86 & 3,93 \\
\hline D7 & 11,07 & 4,68 & 8,83 & 4,03 & 9,66 & 4,50 & 9,20 & 3,66 & 3,33 & 2,79 \\
\hline PA & 10,04 & 4,09 & 9,52 & 4,04 & 10,26 & 4,48 & 10,48 & 4,14 & 4,95 & 3,43 \\
\hline $\mathrm{Pp}$ & 10,49 & 3,91 & 11,51 & 3,56 & 9,68 & 3,60 & 12,54 & 3,56 & 14,31 & 3,49 \\
\hline$S C$ & 8,23 & 4,68 & 8,79 & 3,68 & 8,10 & 4,37 & 9,20 & 4,21 & 3,64 & 2,98 \\
\hline AS & 12,61 & 3,30 & 12,57 & 3,80 & 12,59 & 3,26 & 12,58 & 3,56 & 11,11 & 2,93 \\
\hline PS & 12,53 & 5,54 & 10,28 & 5,55 & 11,89 & 5,57 & 12,61 & 4,68 & 4,33 & 3,37 \\
\hline
\end{tabular}

Nota: $\mathrm{N}$ del grupo normativo = 2646 varones y 325 mujeres. D1= Hipocondríasis, D2= Depresión suicida, D3= Agitación, D4= Depresión ansiosa, D5= Depresion-baja energía, D6= Culpa-resentimiento, D7= Apatía-retirada, $\mathrm{Pa}=$ Paranoia, $\mathrm{Pp}=$ Desviación psicopática, $\mathrm{Sc}=$ Esquizofrenia, As= Psicastenia, $\mathrm{Ps}=$ Desajuste psicológico. 
han obtenido ponderando los valores normativos para hombres y mujeres ofrecidos en la tercera edición del manual del test por TEA (Krug, 1987/1994), según el procedimiento expresado por Garrett (1974).

En la Figura 1 se muestran los perfiles de cada grupo clínico en puntuaciones $\mathrm{T}$.
Estos perfiles se han obtenido con la transformación lineal de las puntuaciones directas medias, tomando como referencia los datos del grupo normativo especificado en la Tabla 2.

La Tabla 3 recoge los coeficientes de consistencia interna para cada una de las

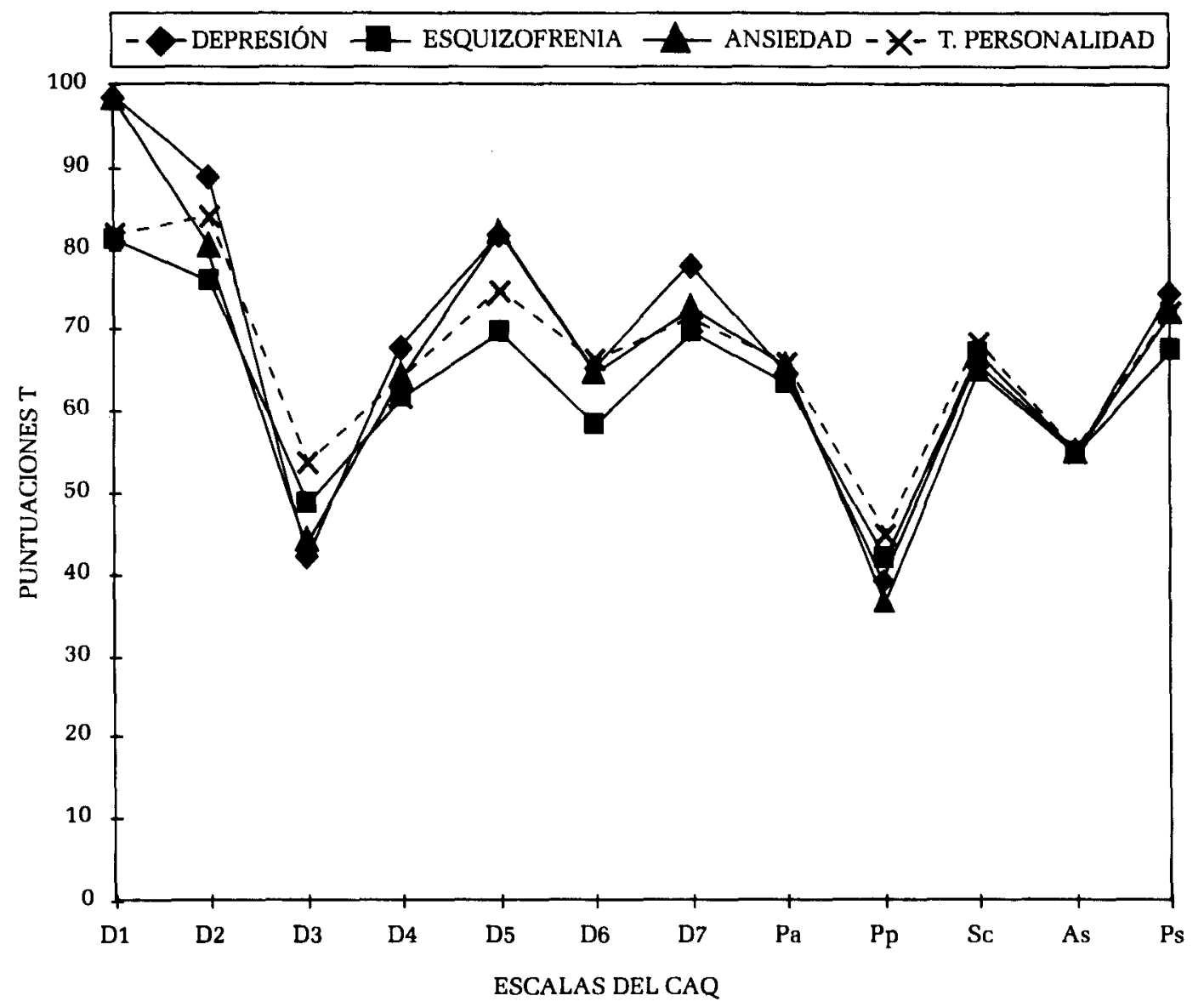

Figura 1. Perfil de los grupos clínicos

D1 = Hipocondríasis, D2 = Depresión suicida, D3= Agitación, D4 = Depresión ansiosa, D5= Depresión-baja energía, $\mathrm{D} 6=$ Culpa-resentimiento, $\mathrm{D} 7=$ Apatfa-retirada, $\mathrm{Pa}=\mathrm{Paranoia}, \mathrm{Pp}=$ Desviación psicopática, $\mathrm{Sc}=$ Esquizofrenia, As= Psicastenia, Ps= Desajuste psicológico.

Tabla 3. Coeficientes de consistencia interna (alfa de Cronbach) para cada una de las escalas del CAQ en la muestra clínica total $(\mathrm{N}=250)$

\begin{tabular}{|c|c|c|c|c|}
\hline \multicolumn{2}{|c|}{ ESCALAS DEL CAQ } & \multirow{2}{*}{$\frac{\alpha}{0,86}$} & ESCALAS DEL CAQ & \multirow{2}{*}{$\frac{\alpha}{0,64}$} \\
\hline D1 & Hipocondríasis & & D7 Apatía-retirada & \\
\hline D2 & Depresión suicida & 0,89 & $\mathrm{~Pa}$ Paranoia & 0,68 \\
\hline D3 & Agitación & 0,42 & Pp Desviación psicopática & 0,56 \\
\hline D4 & Depresión ansiosa & 0,62 & Sc Esquizofrenia & 0,68 \\
\hline D5 & Depresión-baja energía & 0,89 & As Psicastenia & 0,52 \\
\hline D6 & Culpa-resentimiento & 0,83 & Ps Desajuste psicológico & 0,83 \\
\hline
\end{tabular}


escalas del CAQ, calculados a partir de las puntuaciones directas obtenidas por el conjunto de sujetos de la muestra.

Cinco escalas presentan un valor alfa superior a 0,80 (D1 Hipocondriasis, D2 Depresión suicida, D5 Depresión-baja energía, D6 Culpa-resentimiento y Ps Desajuste Psicológico). La escala D3 Agitación es la que muestra menor consistencia interna. El valor medio de alfa obtenido en el presente trabajo es 0,70. Este valor es equivalente al ofrecido por Krug (1980) para la muestra americana con sujetos normales (valor medio de $\alpha=0,71$ ), y es superior al que ofrece la adaptación española (valor medio de $\alpha=0,51$ ). Las escalas D3 (Agitación), D7 (Apatía-retirada) y As (Psicastenia) podrían mejorar su fiabilidad si se suprimieran los ítems 118, 49 y 130, respectivamente, dado que mantienen una correlación negativa con el total de su escala.

Para comprobar el efecto que el diagnóstico, la edad y el sexo pueden tener sobre las puntuaciones obtenidas por los diferentes grupos clínicos en las escalas del CAQ, se ha realizado un análisis factorial de la varianza con tres factores: grupo clínico (depresión, esquizofrenia, ansiedad $\mathrm{y}$ trastornos de personalidad) $\mathrm{x}$ sexo (hombres y mujeres) $x$ edad $(<25$ años; 25-40 años; >40 años).

En la Tabla 4 se especifica el valor $F$ y la probabilidad sólo para aquellas escalas en las que se han encontrado valores significativos en el efecto principal. Se expresan los factores a los que está asociada la variabilidad (grupo clínico, edad y sexo) y los contrastes correspondientes. Como puede observarse, para los grupos clínicos se han hallado diferencias significativas en cinco escalas del'CAQ (D1 Hipocondriasis, D2 Depresión-suicida, D3 Agitación, D4 Depresión ansiosa, D5 Depresión-baja energía y Pp Desviación psicopática). En relación al factor edad se detectan diferencias en tres escalas (D3 Depresión ansiosa, Pp Desviación psicopática y Sc Esquizofrenia) y finalmente, la escala Pp Desviación psicopática ofre-

Tabla 4. Análisis factorial de la varianza: Grupo clínico (4) x Edad (3) x Sexo (2).

Valores $F$ y contrastes Scheffée $(p<0,05)$

\begin{tabular}{|c|c|c|c|c|c|c|c|c|}
\hline \multirow[b]{2}{*}{ ESCALA } & \multicolumn{5}{|c|}{ ANÁLISIS FACTORIAL DE LA VARIANZA } & \multicolumn{3}{|c|}{ CONTRASTES SCHEFFÉE } \\
\hline & & $\begin{array}{c}\text { EFECTO } \\
\text { PRINCIPAL }\end{array}$ & $\begin{array}{l}\text { GRUPOS } \\
\text { CLÍNICOS }\end{array}$ & EDAD & SEXO & GRUPO & EDAD & SEXO \\
\hline $\mathrm{D} 1$ & $\begin{array}{l}F \\
p\end{array}$ & $\begin{array}{c}3,87 \\
<0,001\end{array}$ & $\begin{array}{c}5,82 \\
<0,001\end{array}$ & ns & ns & $\begin{array}{l}D>E \\
D>P \\
A>E \\
A>P\end{array}$ & ns & ns \\
\hline D2 & $\begin{array}{l}F \\
p\end{array}$ & $\begin{array}{c}2,47 \\
<0,025\end{array}$ & $\begin{array}{c}3,27 \\
<0,022\end{array}$ & ns & ns & $\mathrm{D}>\mathrm{E}$ & ns & ns \\
\hline D3 & $\begin{array}{l}F \\
p\end{array}$ & $\begin{array}{c}9,50 \\
<0,001\end{array}$ & $\begin{array}{c}5,20 \\
<0,002\end{array}$ & $\begin{array}{c}7,46 \\
<0,001\end{array}$ & ns & $\begin{array}{l}E>D \\
P>D\end{array}$ & $\begin{array}{l}1>2 \\
1>3\end{array}$ & ns \\
\hline D5 & $\begin{array}{l}F \\
p\end{array}$ & $\begin{array}{r}4,61- \\
<0,001\end{array}$ & $\begin{array}{c}7,94 \\
<0,001\end{array}$ & ns & ns & $\begin{array}{l}\mathrm{P}>\mathrm{A} \\
\mathrm{D}>\mathrm{E} \\
\mathrm{A}>\mathrm{E}\end{array}$ & $\begin{array}{c}2>3 \\
\text { ns }\end{array}$ & ns \\
\hline $\mathrm{Pp}$ & $\begin{array}{l}F \\
p\end{array}$ & $\begin{array}{c}5,18 \\
<0,001\end{array}$ & $\begin{array}{c}3,40 \\
<0,019\end{array}$ & $\begin{array}{r}2,88 \\
<0,05\end{array}$ & $\begin{array}{c}7,11 \\
<0,008\end{array}$ & $\begin{array}{l}E>A \\
P>A\end{array}$ & $1>3$ & $\mathrm{H}>\mathrm{M}$ \\
\hline Sc & $\begin{array}{l}F \\
p\end{array}$ & $\begin{array}{c}4,02 \\
<0,001\end{array}$ & ns & $\begin{array}{l}10,06 \\
<0,001\end{array}$ & ns & ns & $\begin{array}{l}1>2 \\
1>3\end{array}$ & ns \\
\hline
\end{tabular}

Nota: $\mathrm{D}=$ Depresivos, $\mathrm{A}=$ Ansiosos, $\mathrm{E}=$ Esquizofrénicos, $\mathrm{P}=$ Trastornos de Personalidad. EDAD: $1=<25$ años, $2=25-40$ años, $3=>40$ años. SEXO: $M=$ Mujeres, $H=$ Hombres. D1 = Hipocondríasis, D2= Depresión suicida, $\mathrm{D} 3=$ Agitación, D5= Depresión-baja energfa, $\mathrm{Pp}=$ Desviación psicopática, $\mathrm{Sc}=$ Esquizofrenia. 
ce diferencias vinculadas al factor sexo.

La fuente de diferenciación en la escala D1 (Hipocondríasis), se halla vinculada al grupo clínico. Los grupos depresivos y ansiosos obtienen puntuaciones equivalentes entre sí, y significativamente superiores a los grupos de esquizofrénicos y de trastornos de personalidad, los cuales obtienen, también, puntuaciones medias equivalentes entre sí.

El grupo clínico es también la principal fuente de variación en la escala D2 (Depresión suicida). En este caso el grupo de depresivos obtiene puntuaciones superiores al de esquizofrénicos. Los otros dos grupos clínicos obtienen puntuaciones intermedias en relación a los anteriores, sin presentar diferencias significativas respecto a ninguno de ellos.
El grupo clínico y la edad son la fuente de diferenciación en la escala D3 (Agitación). Respecto de la edad, el grupo de sujetos menores de 25 años obtiene puntuaciones más elevadas que los otros dos grupos de edad. Asimismo, el grupo de edad intermedia obtiene puntuaciones más elevadas que el de mayor edad. Las puntuaciones obtenidas en esta escala D3 según edad y grupo clínico se expresa de forma gráfica en la Figura 2. Se observa que, a) el grupo de depresivos jóvenes puntúan por debajo de los demás grupos clínicos; b) en las edades intermedias, el grupo clínico de trastornos de personalidad obtiene la mayor puntuación media, y c) el grupo de depresivos obtiene puntuaciones bajas en los tres grupos de edad.

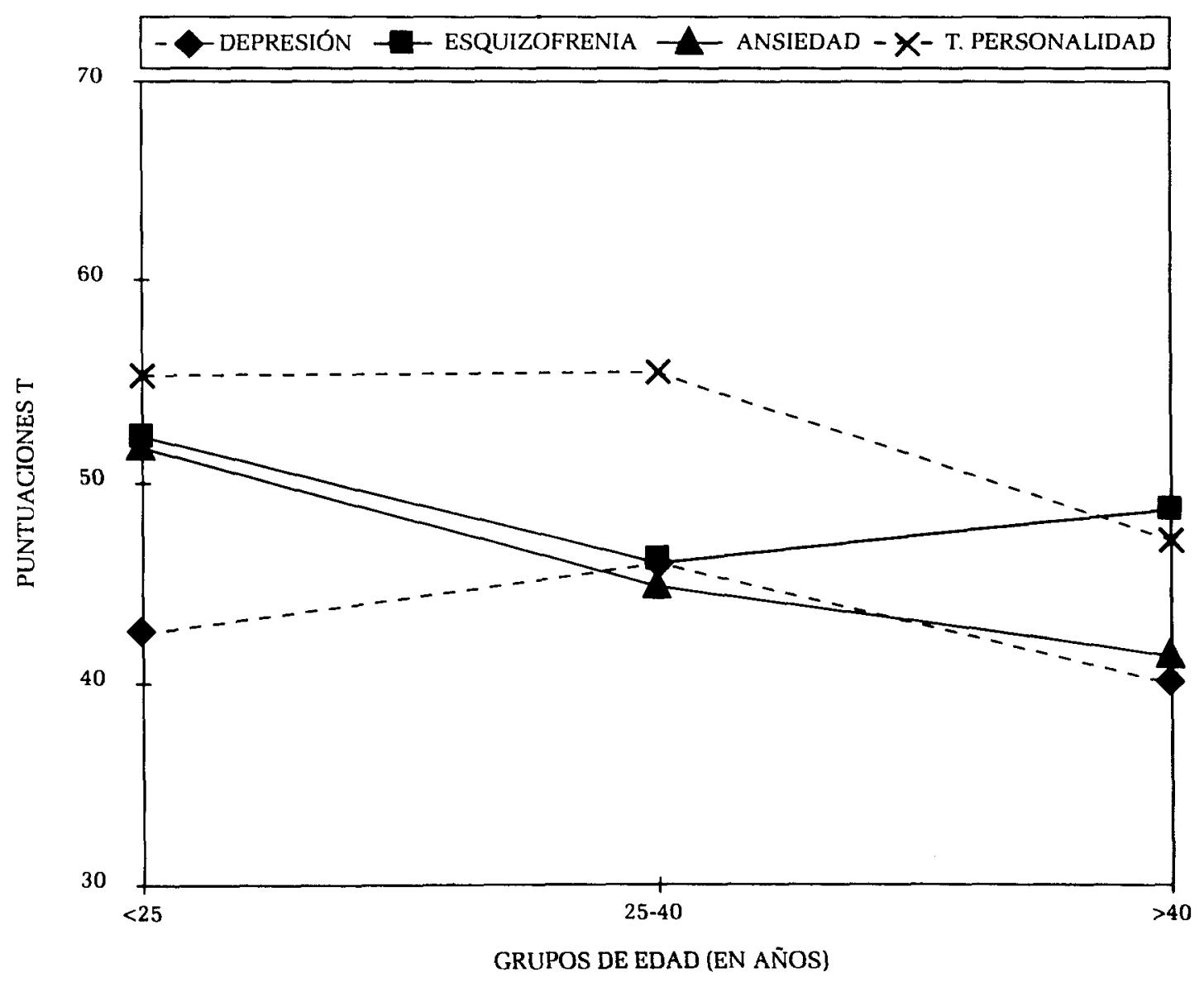

Figura 2. Escala D3, Agitación: Interacción edad × grupo clínico 
El grupo clínico es el principal elemento diferenciador para la escala D5 (Depresión-baja energía). El grupo de esquizofrénicos obtiene la menor puntuación y se diferencia significativamente de los grupos de depresivos y ansiosos, cuyas puntuaciones son equivalentes entre sí. El grupo de sujetos con trastornos de personalidad no se diferencia significativamente de ninguno de los otros tres.

La variabilidad en la escala Pp (Desviación psicopática) se debe tanto a los efectos principales de los tres factores como a su interacción. La interacción sexo $x$ edad, $F(2,212)=3,76, p=0,025$, se expresa en la Figura 3. Las mujeres obtienen mayor puntuación en el grupo joven y los hombres en los grupos de edad intermedia y mayor edad. La puntuación media obtenida por las mujeres desciende a medida que avanza la edad, $F(2,212)=7,83, p<0,001$, mientras que en los hombres no se detectan diferencias significativas debidas a la edad. Los hombres obtienen puntuaciones significativamente más altas que las mujeres en el grupo de edad intermedio, $F(2,212)=8,46$, $p=0,004$, y en el grupo de edad alta, $F(2,212)=4,35, p=0,04$.

La interacción edad $x$ grupo clínico ha resultado también significativa, $F(6,208)=$ 2,55, $p=0,021$, en la escala Pp (Desviación psicopática) tal como aparece en la Tabla 4. Se aprecian diferencias significativas, según el grupo clínico, en los más jóvenes, $F(6,208)=3,35, p=0,03$, siendo el grupo de depresivos el que obtiene las pun-

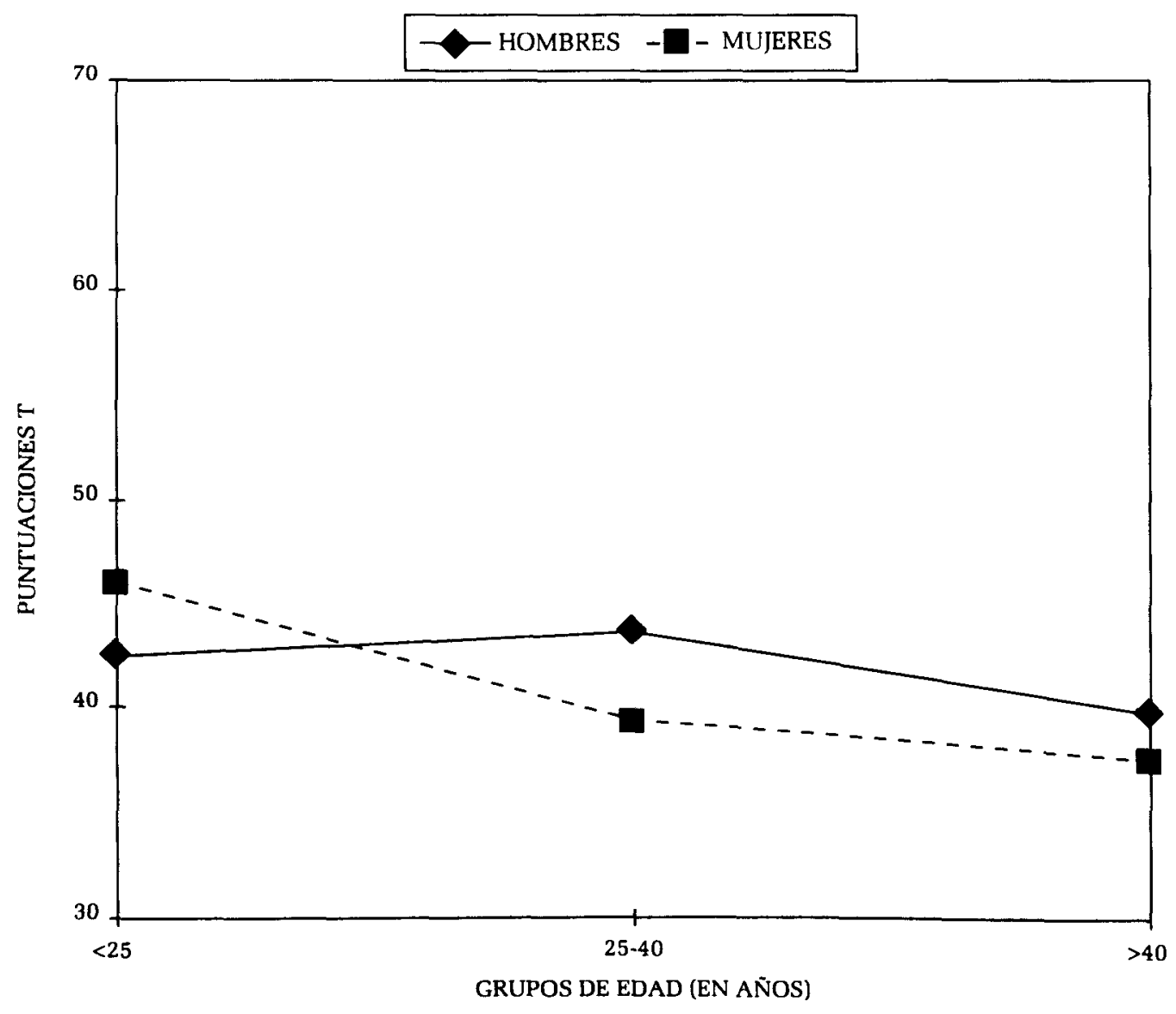

Figura 3. Escala Pp, Desviación psicopática: Interacción sexo $\times$ edad 
tuaciones más bajas. El grupo clínico de trastornos de personalidad mantiene puntuaciones elevadas en los tres grupos de edad. Los grupos de esquizofrénicos y ansiosos tienden a obtener menor puntuación a medida que aumenta la edad.

En la escala Sc (Esquizofrenia) la variabilidad está relacionada con la edad. Los jóvenes obtienen puntuaciones más elevadas que los otros dos grupos de edad, que no se diferencian entre sí.

Se ha llevado a cabo un análisis discriminante con las 5 escalas del CAQ que el ANOVA precedente ha detectado como capaces de diferenciar entre grupos clínicos: D1, Hipocondríasis; D2, Depresión suicida; D3, Agitación; D5, Depresión-baja energía, y Pp, Desviación psicopática.
Este análisis se ha realizado en dos pasos: primero con los cuatro grupos clínicos y después tomando los grupos clínicos dos a dos. En este segundo paso se han excluido los contrastes entre depresivos versus ansiosos y esquizofrénicos versus trastornos de personalidad, ya que el análisis factorial de la varianza no detectó diferencias significativas entre ellos. En la Tabla 5 se presentan los datos correspondientes a estos dos pasos del análisis discriminante. Se especifican los porcentajes de clasificación globales, los porcentajes de casos correctamente clasificados, las escalas que contribuyen significativamente a la discriminación y los valores lambda de Wilks correspondientes.

La matriz de confusión resultante para los cuatro grupos clasifica adecuadamen-

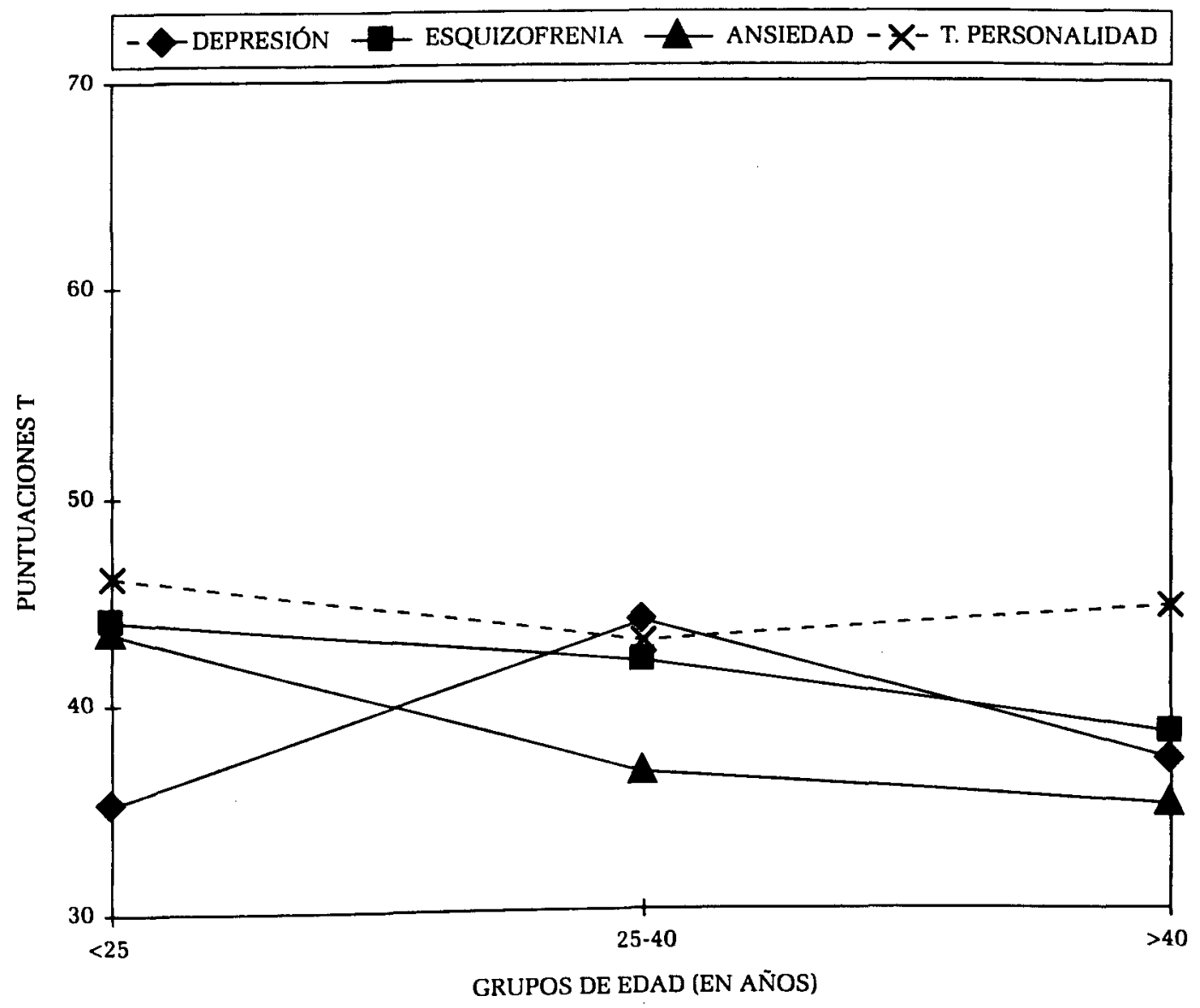

Figura 4. Escala Pp, Desviación psicopática: Interacción edad $\times$ grupo clínico 
Tabla 5. Análisis discriminante de 5 escalas del CAQ y grupos clínicos

\begin{tabular}{lcccccccccc}
\hline & \multicolumn{3}{c}{ \% de casos correctamente clasificados } & \multicolumn{3}{c}{ valores lambda Wilks } \\
\cline { 2 - 10 } & total & dep. & esq. & ans. & t.pers. & D1 & D2 & D3 & D5 & PP \\
\hline 4 grupos clínicos & $47 \%$ & $46 \%$ & $43 \%$ & $46 \%$ & $56 \%$ & 0,001 & & 0,001 & 0,001 & 0,008 \\
Depresivos vs Esquizofrénicos & $67 \%$ & $68 \%$ & $66 \%$ & & & 0,003 & & 0,001 & 0,001 & \\
Depresivos vs T. Personalidad & $77 \%$ & $78 \%$ & & & $75 \%$ & 0,006 & & $\mathbf{0 , 0 0 1}$ & & \\
Esquizofrénicos vs Ansiosos & $70 \%$ & & $66 \%$ & $73 \%$ & & 0,001 & & 0,003 & 0,001 & 0,009 \\
Ansiosos vs T. Personalidad & $73 \%$ & & & $73 \%$ & $73 \%$ & 0,002 & & $\mathbf{0 , 0 0 1}$ & & 0,001 \\
\hline
\end{tabular}

te el $47,4 \%$ de sujetos. Quedan clasificados en el grupo clínico pertinente el $46 \%$ de sujetos depresivos, el $43 \%$ de esquizofrénicos, el $46 \%$ de ansiosos y el $56 \%$ de sujetos con trastornos de personalidad. La escala D2 Depresión suicida no contribuye significativamente a la discriminación

El análisis discriminante entre los grupos depresivos y esquizofrénicos ofrece un valor de clasificación global de $66,9 \%$, y clasifica adecuadamente el $68 \%$ de depresivos y el $66 \%$ de esquizofrénicos. Tres escalas contribuyen significativamente a la discriminación: D1 (Hipocondríasis), D3 (Agitación) y D5 (Depresiónbaja energía).

El porcentaje de clasificación global para los grupos de depresivos y trastornos de personalidad alcanza un $77 \%$, clasificando correctamente el $78 \%$ y el $76 \%$ respectivamente. Las escalas D1 (Hipocondríasis) y D3 (Agitación) contribuyen, significativamente, a la discriminación.

Entre esquizofrénicos y ansiosos, el porcentaje de clasificación global es de $70,1 \%$. Quedan correctamente clasificados el $66 \%$ de esquizofrénicos y el $73 \%$ de ansiosos. Contribuyen a la discriminación las escalas D1 (Hipocondríasis), D3 (Agitación), D5 (Depresión-baja energía) y Pp (Desviación psicopática).

Finalmente, el análisis discriminante entre los grupos de ansiosos y trastornos de personalidad alcanza un valor global de clasificación correcta del $73,1 \%$. Quedan correctamente clasificados el $73 \%$ de ansiosos y el $73 \%$ de sujetos con trastorno de personalidad. Las escalas que intervienen en la clasificación son la D1 (Hipocondríasis), D3 (Agitación) y $\mathrm{Pp}$ (Desviación psicopática).

\section{CONCLUSIONES Y DISCUSIÓN}

En este trabajo se ha pretendido analizar la fiabilidad y el poder discriminante de las escalas del CAQ en población clínica. Se ha obtenido una consistencia interna media de 0,70. Este valor es equivalente al que ofrece la versión original con población normal, y mayor que el obtenido en la versión española, también en población normal. Aunque el valor global de la fiabilidad conseguido en este trabajo puede considerarse satisfactorio, conviene señalar que es insuficiente en algunas escalas. La revisión o supresión de algunos ítems podría mejorar estos coeficientes.

Se ha analizado la utilidad del CAQ para diferenciar entre grupos clínicos. En primer lugar se ha averiguado en qué medida cada una de las escalas permite distinguir entre grupos clínicos, determinando el peso que las variables sexo, edad y diagnóstico tienen en tal diferenciación. En segundo lugar, se ha buscado el valor discriminante global del test.

Según la estructura teórica del CAQ, las siete escalas de depresión deberían sustentar la diferenciación del grupo de depresivos y las restantes deberían per- 
mitir identificar el resto de alteraciones psicopatológicas. Sin embargo, según los datos de este trabajo, sólo tres de las doce escalas del CAQ, Hipocondríasis, Depresión-suicida y Depresión-baja energía, permiten diferenciar específicamente entre algunos de los grupos clínicos establecidos. En estas tres escalas todos los grupos clínicos obtienen puntuaciones que se elevan por encima de la puntuación T 70.

Otras dos escalas, Agitación y Desviación psicopática discriminan entre grupos clínicos cuando se tienen en cuenta, además, variables de edad y sexo. Por ejemplo, la escala Agitación distingue entre grupos clínicos cuando son jóvenes; la escala de Desviación psicopática establece diferencias significativas sólo cuando los sujetos son jóvenes y varones. No obstante, conviene destacar que las puntuaciones $\mathrm{T}$ obtenidas por los grupos clínicos en estas dos escalas se sitúan dentro de la normalidad. Por esta razón y a pesar de las diferencias estadísticas halladas, dichas escalas aportan, a nuestro juicio, una limitada utilidad diagnóstica.

La edad es el único factor diferenciador en la escala de Esquizofrenia, en la cual los grupos clínicos resultan indiferenciados, aunque con una puntuación $\mathrm{T}$ muy próxima al valor 70 .

El resto de escalas, ofrece escaso valor diferenciador entre grupos psicopatológicos.

Globalmente, puede afirmarse que el CAQ no discrimina entre los grupos que hemos establecido de esquizofrénicos y trastornos de personalidad, los cuales presentan un perfil muy similar. Igual ocurre entre los grupos de ansiosos y depresivos.

El conjunto de estos datos coincide, en líneas generales, con los obtenidos por Martorell et al. (1995) que ponen de manifiesto que el CAQ, si bien es sensible a la presencia de alteración psicopa- tológica, no parece ser un instrumento suficientemente apropiado para el diagnóstico diferencial de psicopatología. No obstante, antes de concluir acerca de la falta de validez discriminante, convendría replantear el análisis discriminante con grupos clínicos con mayor número de sujetos y, sobre todo, más específicos en cuanto a diagnóstico psicopatológico.

\section{REFERENCIAS}

Abad, J., Amador, J.A., Forns, M. y Martorell, B. (1996). Análisis de la estructura factorial del CAQ en población adolescente. Revista Iberoamericana de Diagnóstico y Evaluación Psicológica, 2, 23-41.

APA (1987). DSM-III-R. Manual diagnóstico y estadístico de los trastornos mentales, revisado. Barcelona: Masson

Boyle, G.J. (1987). Psychopathological depression superfactors measured in the clinical analysis questionnaire. Personality and Individual Differences, 8, 609-614.

Carpenter, D.R. (1995). Adult Children of Alcoholics: CAQ Profiles. Alcoholims Treatment Quarterly, 13, 63-70.

Cattell, R.B. (1973). A check on the 28 factor Clinical Analysis Questionnaire structure on normal and pathological subjects. Journal of Multivariate Experimental Personality and Clinical Psychology, 1, 3-12.

Cattell, R.B. (1975). 16PF. Cuestionario de Personalidad. Formas $A, B$ y $C$. Madrid: TEA.

Cattell, R.B. y Bjerstedt, A. (1967) The structure of depression by factoring $\mathrm{Q}$-data, in relation to general personality source traits. Scandinavian Journal of Psychology, 8, 1724.

Cattell, R.B. y Bolton, L.S. (1969). What pathological dimensions lie beyond the normal dimensions of the 16 PF? A comparison of MMPI and 16PF factors domains. Journal of Consulting and Clinical Psychology, 33, 18-29.

Delhees, K. H. y Cattell, R. B.(1971). The dimensions of pathology: Proof of their projection beyond the normal 16PF source traits. Personality, 2, 461-467. 
Garrett, H.E. (1974). Estadística en psicología y educación. Buenos Aires: Paidós.

Kameoka, V.A. (1986). The structure of the clinical analysis questionnaire and depression symptomatology. Multivariate Experimental Clinical Research, 21, 105-122.

Krug, S. (1980). Clinical Analysis Questionnaire, Manual. Champaign, ILL: Institute for Personality and Ability Testing.

Krug, S. (1987/1994). CAQ. Cuestionario de Análisis Clínico. Madrid: TEA.

Krug, S. (1989). Linear T score norms for the clinical analysis questionnaire. Multivariate Experimental Clinical Research, 92, 1-9.

Krug, S. y Cattell, R. B. (1980). Clinical Analysis Questionnaire Manual. Champaign, ILL: Institute for Personality and Ability Testing.

Marcos, T. y Carné, R.M. (1988). Factores normales y anormales de personalidad en pacientes con agorafobia con crisis de angustia. Revista de Psiquiatría de la Facultad de Medicina de la Universidad de Barcelona, 15, 179-185.
Martorell, B., Amador, J.A. y Forns M. (1994). Estructura factorial exploratoria del HSPQ y del CAQ en población adolescente. Psicothema, 6, 207-214.

Martorell, B., Forns, M., Amador, J.A. y Abad, J. (1995). 16PF y CAQ: perfiles diferenciales en población clínica española. Revista de Psiquiatría de la Facultad de Medicina de la Universidad de Barcelona, 22, 72-79.

Montag, I. y Birenbaum, M. (1988). On the relationship between the MMPI and Cattell's normal and abnormal Personality Factors. Multivariate Experimental Clinical Research, 8, 275-286.

Schwartz, S.A. (1976). Second order factors of the CAQ in a normal population. Multivariate Experimental Clinical Research, 2, 167-172.

Sirigatti, S., Stefanile, C. y Giannetti, E. (1990). Il Clinical Analysis Questionnaire (CAQ): adattamento preliminare per l'Italia. Bolletino di Psicologia Applicata, 195, 3-13. 